International Journal of Theoretical and Applied Finance

Vol. 17, No. 8 (2014) 1499001 (4 pages)

(C) World Scientific Publishing Company

DOI: $10.1142 / \mathrm{S} 0219024914990015$

\title{
Author Index Volume 17 (2014)
}

Abbas-Turki, L. A. \& Lamberton, D., European Options Sensitivity with Respect to the Correlation for Multidimensional Heston Models

Andresen, A., Benth, F. E., Koekebakker, S. \& Zakamulin, V., The CARMA Interest Rate Model

Ankirchner, S., Pigorsch, C. \& Schweizer, N., Estimating Residual Hedging Risk with Least-Squares Monte Carlo

Appolloni, E., Gaudenzi, M. \& Zanette, A., The Binomial Interpolated Lattice Method for Step Double Barrier Options

Astic, F. \& Tourin, A., On the Credit Risk of Secured Loans with Maximum Loan-toValue Covenants

Bayer, C. \& Veliyev, B., Utility Maximization in a Binomial Model with Transaction Costs: A Duality Approach Based on the Shadow Price Process

Benth, F. E., see Andresen, A.

Bermin, H.-P., On Dynamic Forward Rate Modeling and Principal Component Analysis

Beveridge, C. \& Joshi, M., The Efficient Computation of Prices and Greeks for Callable Range Accruals Using the Displaced-Diffusion LMM
3 (2014) 1450015 Buryak, A. \& Guo, I., Effective and Simple VWAP Options Pricing Model

Capriotti, L., see

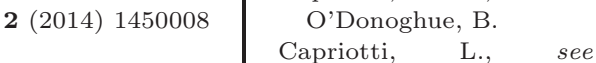

Stehlíková, B. 6 (2014) 1450037

Chen, T.-Y., see Han, C.$\mathrm{H}$.

$7(2014) 1450042$

Cordero, F., Klein, I.
\& Perez-Ostafe, L., Binary Markets Under Transaction Costs

Cox, A. M. G., Hobson, D. \& Obłój, J., Utility

6 (2014) $1450035 \quad$ Theory Front to Back — Inferring Utility from Agents' Choices

Dang-Nguyen, S., Le Caillec, J.-M. \& Hillion, A., Calibration of the UniVariate Cox-IngersollRoss Model and Parameters Selection Through the Kullback-Leibler Divergence Q., Kandhai, D. \& Oosterlee, C. W., Efficient Computation of Exposure Profiles for Counterparty Credit Risk

5 (2014) 1450029 Di Nunno, G. \& Sjursen, S., Information and Optimal Investment in Defaultable Assets

Dorfleitner, G. \& Pfister, T., Justification of Per-Unit Risk Capital
3 (2014) 1450016

4 (2014) 1450023

6 (2014) 1450036

3 (2014) 1450017

2 (2014) 1450009

5 (2014) 1450030

3 (2014) 1450018

6 (2014) 1450038

4 (2014) 1450024

8 (2014) 1450050

1 (2014) 1450001 
Allocation in Portfolio Credit Risk Models

Dyrssen, H., Ekström, E. \& Tysk, J., Pricing Equations in Jump-toDefault Models

Ekström, E., see Dyrssen, $\mathrm{H}$.

Elliott, R. J. \& Hamada, A. S., Option Pricing Using a Regime Switching Stochastic Discount Factor

Feng, Q., see de Graaf, C. S. L.

Fouque, J.-P., Saporito, Y. F. \& Zubelli, J. P., Multiscale Stochastic Volatility Model for Derivatives on Futures

Frey, R. \& Rösler, L., Contagion Effects and Collateralized Credit Value Adjustments for Credit Default Swaps

Fukasawa, M., Volatility Derivatives and ModelFree Implied Leverage

Gatumel, M. \& Ielpo, F., The Number of Regimes Across Asset Returns: Identification and Economic Value

Gaudenzi, M., see Appolloni, E.

Gobet, E. \& Hok, J., Expansion Formulas for Bivariate Payoffs with Application to Best-of Options on Equity and Inflation

Gonon, L. \& Rogers, L. C. G., Evolution of Firm Size

Grzelak, L. A., see van der Stoep, A. W.

Guo, I., see Buryak, A.

Hainaut, D. \& Robert, C. Y., Credit Risk Valuation with Rating Transitions and Partial Information

Hamada, A. S., see Elliott, R. J.

Han, C.-H., Liu, W.H. \& Chen, T.-Y. VaR/CVaR Estimation Under Stochastic Volatility Models

Härtel, M., see Biagini, F.
6 (2014) 1450039

3 (2014) 1450019

3 (2014) 1450019

3 (2014) 1450020

4 (2014) 1450024

7 (2014) 1450043

7 (2014) 1450044

1 (2014) 1450002

6 (2014) 1450040

6 (2014) 1450035

2 (2014) 1450010

5 (2014) 1450031

7 (2014) 1450045

6 (2014) 1450036

7 (2014) 1450046

3 (2014) 1450020

2 (2014) 1450009

3 (2014) 1450016
Heuwelyckx, F., Convergence of European Lookback Options with Floating Strike in the Binomial Model

Hillion, A., see DangNguyen, S.

Hobson, D., see Cox, A. M. G.

Hok, J., see Gobet, E.

Ielpo, F., see Gatumel, M.

Joshi, M., see Beveridge, C.

Kandhai, D., see de Graaf, C. S. L.

Kijima, M. \& Siu, C. C., Credit-Equity Modeling Under a Latent Lévy Firm Process

Klein, I., see Cordero, F.

Koekebakker, S., see Andresen, A.

Kovacevic, R. M. \& Pflug, G. C., Are Time Consistent Valuations Information Monotone?

Kromer, E. \& Overbeck, L., Representation of BSDE-Based Dynamic Risk Measures and Dynamic Capital Allocations

Lamberton, D., see Abbas-Turki, L. A.

Le Caillec, J.-M., see Dang-Nguyen, S.

Lee, J., see O'Donoghue, B.

Lépinette, E., see Tahar, I. B.

Levendorskii, S., Method of Paired Contours and Pricing Barrier Options and CDSs of Long Maturities

Li, M. \& Mercurio, F., Closed-Form Approximation of Perpetual Timer Option Prices

Liu, R. H., A FiniteHorizon Optimal Investment and Consumption Problem Using RegimeSwitching Models

Liu, W.-H., see Han, C.$\mathrm{H}$.

Lo, C. C. \& Skindilias, K., An Improved Markov Chain Approximation
4 (2014) 1450025

6 (2014) 1450038

3 (2014) 1450018

2 (2014) 1450010

6 (2014) 1450040

1 (2014) 1450001

4 (2014) 1450024

3 (2014) 1450021

5 (2014) 1450030

2 (2014) 1450008

1 (2014) 1450003

5 (2014) 1450032

3 (2014) 1450015

6 (2014) 1450038

3 (2014) 1450017

2 (2014) 1450011

5 (2014) 1450033

4 (2014) 1450026

4 (2014) 1450027

2 (2014) 1450009 
Methodology: Derivatives Pricing and Model Calibration

Löhne, A. \& Rudloff, B., An Algorithm for Calculating the Set of Superhedging Portfolios in Markets with Transaction Costs

Lütkebohmert, E. \& Matchie, L., Value-atRisk Computations in Stochastic Volatility Models Using SecondOrder Weak Approximation Schemes

Lütkebohmert, E., see von Hammerstein, E. A.

Macrina, A., Heat Kernel Models for Asset Pricing

Madan, D. B. \& Schoutens, W., Two Processes for Two Prices

Matchie, L., see Lütkebohmert, E.

Mercurio, F., see Li, M.

Nyström, K., Ould Aly, S. M. \& Zhang, C., Market Making and Portfolio Liquidation Under Uncertainty

Obłój, J., see Cox, A. M. G.

O'Donoghue, B., Peacock, M., Lee, J. \& Capriotti, L., A SpreadReturn Mean-Reverting Model for Credit Spread Dynamics

Oosterlee, C. W., see de Graaf, C. S. L.

Oosterlee, C. W., see van der Stoep, A. W.

Ould Aly, S. M., see Nyström, K.

Overbeck, L., see Kromer, E.

Papin, T. \& Turinici, G., Prepayment Option of a Perpetual Corporate Loan: The Impact of the Funding Costs

Peacock, M., see O'Donoghue, B.

Perez-Ostafe, L., see Cordero, F.

Pfister, T., see Dorfleitner, G.
Pflug, G. C., see Kovacevic, R. M.

7 (2014) $1450047 \quad$ Pigorsch, C., see Ankirchner, $\mathrm{S}$.

Pucci, M., Constant Maturity Treasury Convexity Correction

Rambaud, S. C., A New

2 (2014) $1450012 \quad$ Argument in Favor of Hyperbolic Discounting in Very Long Term Project Appraisal

Robert, C. Y., see Hainaut, D. Gonon, L.

Rösler, L., see Frey, R.

6 (2014) 1450041

7 (2014) 1450048

1 (2014) 1450005

1 (2014) 1450004

4 (2014) 1450026

5 (2014) 1450034

3 (2014) 1450018

3 (2014) 1450017

4 (2014) 1450024

7 (2014) 1450045

5 (2014) 1450034

5 (2014) 1450032

4 (2014) 1450028

3 (2014) 1450017

5 (2014) 1450030

6 (2014) 1450039
Roux, A. \& Zastawniak, with Gradual Exercise under Proportional Transaction Costs

Rudloff, B., see Löhne, A.

Rüschendorf, L., see von Hammerstein, E. A.

Sachs, E. W. \& Schneider, M., Reduced-Order Models for the Implied Variance Under Local Volatility

Salvi, G. \& Swishchuk, A. V., Covariance and Correlation Swaps for Financial Markets with Markov-Modulated Volatilities

Saporito, Y. F., see Fouque, J.-P.

Schneider, M., see Sachs, E. W.

Schoutens, W., see Madan, D. B.

Schweizer, N., see Ankirchner, S.

Siu, C. C., see Kijima, M.

Six, P., On the Shape of Risk Aversion and Asset Allocation

Sjursen, S., see Di Nunno, G.

Skindilias, K., see Lo, C. C.

Stehlíková, B. \& Capriotti, L., An Effective Approximation for Zero-Coupon Bonds and Arrow-Debreu Prices in the BlackKarasinski Model

Surya, B. A. \& Yamazaki, K., Optimal Capital T., American Options
1 (2014) 1450003

7 (2014) 1450042

8 (2014) 1450051

8 (2014) 1450049

7 (2014) 1450046

5 (2014) 1450031

7 (2014) 1450044

8 (2014) 1450052

2 (2014) 1450012

6 (2014) 1450041

8 (2014) 1450053

1 (2014) 1450006

7 (2014) 1450043

8 (2014) 1450053

1 (2014) 1450005

7 (2014) 1450042

3 (2014) 1450021

8 (2014) 1450054

8 (2014) 1450050

7 (2014) 1450047

6 (2014) 1450037 
Structure with Scale Effects Under Spectrally Negative Lévy Models

Swishchuk, A. V., see Salvi, G.

Tahar, I. B. \& Lépinette, E., Vector-Valued Coherent Risk Measure Processes

Tanaka, H. \& Yamada, T., Strong Convergence for Euler-Maruyama and Milstein Schemes with Asymptotic Method

Tassinari, G. L. \& Bianchi, M. L., Calibrating the Smile with Multivariate TimeChanged Brownian Motion and the Esscher Transform

Tourin, A., see Astic, F.

Turinici, G., see Papin, T. Tysk, J., see Dyrssen, H. van der Stoep, A. W., Grzelak, L. A. \& Oosterlee, C. W., The Heston Stochastic-Local Volatility Model: Efficient Monte Carlo Simulation
2 (2014) 1450013

1 (2014) 1450006

2 (2014) 1450011

2 (2014) 1450014

4 (2014) 1450023

8 (2014) 1450055

4 (2014) 1450028

3 (2014) 1450019

7 (2014) 1450045
Veliyev, B., see Bayer, C. von Hammerstein, E. A., Lütkebohmert, E.,

4 (2014) 1450022 Rüschendorf, L. \& Wolf, V., Optimality of Payoffs in Lévy Models

Wolf, V., see von Hammerstein, E. A.

$\mathrm{Xu}$, G. \& Zheng, H., Lower Bound Approximation to Basket Option Values for Local Volatility JumpDiffusion Models

Yamada, T., see Tanaka, H.

Yamazaki, K., see Surya, B. A.

Zakamulin, V., see Andresen, A.

Zanette, A., see Appolloni, E.

Zastawniak, T., see Roux, A.

Zhang, C., see Nyström, K.

Zheng, H., see $\mathrm{Xu}, \mathrm{G}$.

Zubelli, J. P., see Fouque, J.-P.
6 (2014) 1450041

6 (2014) 1450041

1 (2014) 1450007

2 (2014) 1450014

2 (2014) 1450013

2 (2014) 1450008

6 (2014) 1450035

8 (2014) 1450052

5 (2014) 1450034

1 (2014) 1450007

7 (2014) 1450043 